\title{
Journal of Hepatology and

\section{Liver Injury due to Amoxicillin vs. Amoxicillin/Clavulanate: A Subgroup Analysis of a Drug-Induced Liver Injury Case-Control Study in Italy}

Monia Donati ${ }^{1}$, Domenico Motola ${ }^{1}$, Roberto Leone ${ }^{2^{*}}$, Ugo Moretti ${ }^{2}$, Giovanna Stoppa ${ }^{2}$, Elena Arzenton ${ }^{2}$, Maria Carmela Lenti ${ }^{3}$, Roberto Bonaiuti $^{3}$, Alessandro Mugelli $^{3}$, Alfredo Vannacci ${ }^{3}$, Concetta Rafaniello ${ }^{4}$, Liberata Sportiello ${ }^{4}$, Annalisa Capuano ${ }^{4}$, Oscar Bortolami ${ }^{5}$ and Alberto Vaccheri ${ }^{1}$

${ }^{1}$ Unit of Pharmacology, Department of Medical and Surgical Sciences, University of Bologna, Bologna, Italy

${ }^{2}$ Pharmacology Unit, Department of Diagnostics and Public Health, University of Verona, Verona, Italy

${ }^{3}$ Department of Neurosciences, Psychology, Drug Research and Child Health (NeuroFarBa), Tuscan Regional Centre of Pharmacovigilance, University of Florence, Florence, Italy

${ }^{4}$ Pharmacology Unit, Department of Experimental Medicine, Second University of Naples, Naples, Italy

${ }^{5}$ Clinical Trials Research Unit, School of Health and Related Research, University of Sheffield, Sheffield, UK

"Corresponding author: Roberto Leone, Department of Diagnostics and Public Health, University of Verona, p.le L.A. Scuro, 1037134, Verona, Italy, Tel: +39-045-8124706; E-mail: roberto.leone@univr.it

Received date: December 23, 2016; Accepted date: January 17, 2017; Published date: January 20, 2017

Copyright: (C) 2017 Donati M, et al. This is an open-access article distributed under the terms of the Creative Commons Attribution License, which permits unrestricted use, distribution, and reproduction in any medium, provided the original author and source are credited.

\section{Abstract}

Objective: Several studies showed that amoxicillin plus clavulanic acid (co-amoxiclav) is one of the most common agents associated to serious Drug Induced Liver Injury (DILI). We estimated the risk of acute serious DILI associated with amoxicillin alone compared with co-amoxiclav, through a multicenter case-control study carried out in nine Italian hospitals from October 2010 to January 2014.

Methods: Cases were adults, with a diagnosis of acute liver injury. Controls presented acute clinical disorders, not related to chronic conditions and not involving the liver. Adjusted Odds Ratio (ORs) with $95 \% \mathrm{Cl}$ were calculated initially with a bivariate and then multivariate analysis.

Results: We analysed 179 cases matched to 1770 controls. Seven cases were exposed to amoxicillin (adjusted OR $1.69,95 \% \mathrm{Cl} 0.72-3.98$ ) and 22 cases to co-amoxiclav (adjusted OR $3.00,95 \% \mathrm{Cl} 1.76-5.40$ ).

Conclusions: Co-amoxiclav almost doubled the risk of serious acute liver injury compared to amoxicillin alone. The incidence of co-amoxiclav induced DILI is very low but the widespread use of this drug by the general population makes the risk clinically relevant. The often inappropriate prescription of antimicrobial agents, and in particular of co-amoxiclav, could expose a given patient to a life-threatening risk compared to a negligible clinical benefit.

Keywords: Adverse drug reaction; Case-control study; Pharmacovigilance; Pharmacoepidemiology Beta-lactams; Antibiotics; Liver damage

\section{Introduction}

Beta-lactams are the most commonly prescribed antibacterial agents, with large variability across geographical areas [1]. Amoxicillin is used to treat infections caused by susceptible bacteria, such as Escherichia coli, Haemophilus influenzae, Staphylococcus aureus (non-penicillinase producing) and Streptococcus pneumoniae; its combination with clavulanic acid (co-amoxiclav), a potent inhibitor of many bacterial beta-lactamase enzymes, broadens its antimicrobial spectrum. These drugs have been widely used in the outpatient setting for the treatment of community-acquired respiratory tract and other mild-to-moderate infections and in recent years an increased consumption of co-amoxiclav compared to amoxicillin alone has been observed [1].

Several studies showed that co-amoxiclav is one of the most common agents associated to severe drug induced liver injury (DILI) [2-4] and multiple case reports of co-amoxiclav hepatotoxicity have been published since its first marketing [5,6]. In both Latin American and Spanish registries of DILI co-amoxiclav ranks first among drugs involved in liver damage [7]. The liver injuy is reported often as secondary to hypersensitivity drug allergy resulting in centrizonal cholestasis, progressing to hepatocellular damage. In severe reactions, bile duct injury has been reported. The liver injury was mainly related to the clavulanic acid component; as a matter of fact, there are only few reports of hepatic reactions with amoxicillin alone [8-10]. Furthermore, the role of clavulanic acid at the onset of liver damage is supported by data on positive rechallenge (second episode of hepatitis) only after re-administration of co-amoxiclav and not after amoxicillin alone [11]. However, a limited number of studies are available in scientific literature in which a direct comparison between amoxicillin and co-amoxiclav was made. Rodríguez et al. [12], conducted a retrospective cohort study in the United Kingdom in order to estimate the risk of acute liver injury associated with the combination of amoxicillin and clavulanic acid and to compare it with the risk related to amoxicillin alone. Salvo et al. [13] analyzed the Italian database of spontaneous reporting of suspected adverse drug reactions (ADRs) in order to compare the safety profile of these two drugs. Even though 
Page 2 of 5

applying different methodologies, both studies found that coamoxiclav is more hepatotoxic than amoxicillin alone.

Here we present the results of the Drug-Induced Liver Injury casecontrol study in Italy (DILI-IT) [14] concerning the pre-specified subgroup analysis on the risk of acute serious liver injury associated to amoxicillin and co-amoxiclav.

\section{Methods}

The DILI-IT study recruited cases and controls among nine hospitals located in four Italian regions, from October 2010 to January 2014. A detailed description of the methods has already been published elsewhere [14]

We used the following criteria for case definition: all patients aged 18 or over, admitted with a primary diagnosis of acute liver injury according to an increase of $2 \mathrm{~N}$ ( $\mathrm{N}$ is the upper limit of normal range and each activity is expressed as a multiple of $\mathrm{N}$ ) for alanine aminotransferase (ALT), aspartate aminotransferase (AST) serum activity in patients who presented symptoms or not and/or an increase of $1.5 \mathrm{~N}$ of alkaline phosphatase associated with an increase of ALT or AST and/or total bilirubin in patients with or without symptoms $[15,16]$.

Controls were patients with a minimum age of 18 years, admitted for acute clinical disorders not related to chronic conditions, not involving the liver (with normal liver profile values). Ten hospital controls were selected consecutively for each case, matched according to gender and age (+/- 5 years), and to hospital and time from admission (within 2 months). Both cases and controls were excluded if the patients had a diagnosis of viral hepatitis, biliary abnormality, history of alcohol abuse, autoimmune disease, genetic and metabolic disorders which may determine liver injury, a low alpha-1-antitrypsin level and an abnormal phenotype (that may suggest disease associated with a deficiency of this protein), Wilson's disease, HIV/AIDS, hepatic neoplasia or liver metastasis, mushroom poisoning and drug addiction or detoxification treatment in the last 3 months. According to primary exclusion criteria, patients who were not resident in the study areas were also excluded. Finally, patients who were discharged or died before interview and those refused the interview or were unable to answer were also excluded (secondary exclusion criteria).

Exposure to drugs was defined as any use in the 90 days prior to the index day (onset day of the liver damage symptoms or the date corresponding to the first available abnormal value of liver enzyme tests) [16] and was investigated in a face to face interview using a standardized questionnaire. Data concerning the diagnosis of liver injury and the evolution of the disease were collected from medical records. Clinical records were also used to evaluate the diagnoses of the controls.

Statistical analysis of data provided for the calculation of Odds Ratios (OR) and their 95\% confidence intervals (95\% CI), by means of a conditional logistic regression for matched case-control groups, were made using the Stata Statistical Software version 11.0 (StataCorp 2009). In order to assure that the risk estimates for liver injury and use of drugs were appropriately adjusted for possible confounders and effect modifiers, OR has been computed initially with a bivariate and then multivariate analysis. Covariates included were: smoking, alcohol, body mass index (BMI), liver comorbidities (hepatitis, gallstones, liver cirrhosis, hepatic nodules and other hepatic diseases), heart comorbidities (stroke, angina pectoris, heart failure, arrhythmia and cardiac surgery), and concomitant drugs (NSAIDs, paracetamol, macrolides, antidepressants and statins).

The study was approved by the Independent Ethics Committee (IEC) of Verona Hospital (principal investigator and coordinating centre) and consecutively notified to the IECs of participating centres.

\section{Results}

Overall, 2232 patients with diagnosis of acute serious liver injury were recorded in DILI-IT Study; of those, 2028 were not included in the study, according to the primary exclusion criteria. Subsequently, other 25 cases were excluded on the basis of secondary criteria. Therefore, the analysis was performed on 179 cases. In the same period, 3059 patients were selected and interviewed as possible controls. A study flow chart is available in the DILI-IT Study manuscript [14]. From this sample, 1770 patients were matched to the 179 cases, according to gender, age, centre, and time of admission. Controls included in the analysis were enrolled for the following diagnosis: trauma or fracture (46\%), acute appendicitis (15\%), gastrointestinal disorders (14\%), respiratory disorders (13\%), other acute events (12\%). Except for drugs consumption (cases used more drugs than controls), there were no significant differences between cases and controls relating to BMI, school level, alcohol consumption and tobacco use, cardiovascular and hepatic concomitant disease.

During the three months before the index day, 7 cases (4\% of 179) were exposed to amoxicillin and $22(12 \%)$ to co-amoxiclav, resulting in an annual incidence of 0.1 and 0.4 cases per 100,000 inhabitants, respectively. In the same period, 37 controls ( $2 \%$ of the 1770 ) were exposed to amoxicillin and $63(4 \%)$ to co-amoxiclav. Table 1 shows the baseline characteristics of these patients. Also these subgroups of patients did not reveal any significant differences between cases and controls, except for drug consumption.

\begin{tabular}{|l|l|l|l|l|l|l|}
\hline & \multicolumn{2}{l|}{ Amoxicillin } & \multicolumn{2}{l|}{ Co-amoxiclav } \\
\hline Characteristic & Cases $\mathbf{n = 7}$ & Controls n=37 & P value & Cases n=22 & Controls n=63 & P value \\
\hline Sex, $\mathbf{n}(\%)$ & & & 0.09 & & & \\
\hline Male & $0(0)$ & $13(35)$ & & $13(59)$ & $27(43)$ & 0.14 \\
\hline Female & $7(100)$ & $24(65)$ & & $9(41)$ & $36(57)$ \\
\hline Age (years), mean \pm sd & $42 \pm 25.8$ & $45.6 \pm 18.9$ & 0.67 & $44.7 \pm 17.8$ & $42.8 \pm 16.4$ & 0.65 \\
\hline Schooling level (years), mean \pm sd & $12.1 \pm 6.2$ & $11.6 \pm 4.5$ & 0.8 & $11.1 \pm 4.7^{*}$ & $10.4 \pm 4.3^{*}$ & 0.52 \\
\hline
\end{tabular}




\begin{tabular}{|c|c|c|c|c|c|c|}
\hline BMI, mean \pm sd & $24.1 \pm 6.8$ & $24.6 \pm 3.6$ & 0.77 & $24.6 \pm 3.6$ & $24.7 \pm 4.0$ & 0.92 \\
\hline Alcohol, n (\%) & & & 0.99 & & & 0.51 \\
\hline Current drinker & $4(57)$ & $19(51)$ & & $16(73)$ & $39(62)^{\star \star}$ & \\
\hline Former drinker & $0(0)$ & $1(3)$ & & $1(4)$ & $2(3)$ & \\
\hline Non-drinker & $3(43)$ & $17(46)$ & & $5(23)$ & $21(33)$ & \\
\hline Smoke, n (\%) & & & 0.17 & & & 0.99 \\
\hline Current smoker & $3(43)$ & $5(14)$ & & $7(32)$ & $21(33)$ & \\
\hline Former smoker & $1(34)$ & $6(16)$ & & $3(14)$ & $8(13)$ & \\
\hline Non-smoker & $3(43)$ & $26(70)$ & & $12(54)$ & $34(54)$ & \\
\hline \multicolumn{7}{|l|}{ Co-morbidities, n (\%) } \\
\hline Liver diseases & $0(0)$ & $4(11)$ & 0.99 & $3(14)$ & $7(11)$ & 0.71 \\
\hline Heart diseases & $2(29)$ & $4(11)$ & 0.99 & $2(9)$ & $5(8)$ & 0.99 \\
\hline Number of drugs, mean \pm sd & $6.9 \pm 3.3$ & $3.6 \pm 1.6$ & $<0.001$ & $4.9 \pm 2.7$ & $3.6 \pm 2.4$ & 0.04 \\
\hline
\end{tabular}

Table 1: Baseline characteristics of patients in study population.

About $60 \%$ of cases taking co-amoxiclav were men, while $100 \%$ of cases exposed to amoxicillin were women; average age, school level and BMI in the two groups were approximately the same. Concomitant cardiovascular diseases were more frequent in cases taking amoxicillin (29\%), than in cases taking co-amoxiclav (9\%); on the contrary, concomitant liver diseases were present only in the latter ( 3 cases of light steatosis). Cases treated with amoxicillin alone used more drugs than the co-amoxiclav group $(6.9 \pm 3.3$ and $4.9 \pm 2.7$ respectively), however the percentages of patients with concomitant known hepatotoxic drugs were similar.

Table 2 shows the OR estimates of acute serious liver injury associated with amoxicillin and co-amoxiclav: only the association with clavulanic acid was associated to a statistically significant increased risk of liver damage, with an adjusted OR equal to 3.00 (95\% CI, 1.76-5.40). The nature of liver enzyme abnormality was mainly cholestatic in both groups.

\begin{tabular}{|l|l|l|l|l|}
\hline Drug & $\begin{array}{l}\text { Cases } \\
(\mathbf{n}=\mathbf{1 7 9})\end{array}$ & $\begin{array}{l}\text { Controls } \\
\mathbf{( n = 1 7 7 0 )}\end{array}$ & $\begin{array}{l}\text { OR (95\% } \\
(\mathbf{n = 1 9 4 8 )}\end{array}$ & $\begin{array}{l}\text { ORADJ (95\% Cl) } \\
(\mathbf{n}=\mathbf{1 9 3 3 )}\end{array}$ \\
\hline Amoxicillin & $7(4 \%)$ & $37(2 \%)$ & $1.94(0.85-4.44)$ & $1.69(0.72-3.98)$ \\
\hline $\begin{array}{l}\text { Co- } \\
\text { amoxiclav }\end{array}$ & $22(12 \%)$ & $63(4 \%)$ & $3.08(2.42-7.05)$ & $3.00(1.76-5.40)$ \\
\hline
\end{tabular}

Table 2: Risk of acute serious drug-induced liver injury associated with amoxicillin and amoxicillin/clavulanic acid.

\section{Discussion}

Our data show that the combination of amoxicillin with clavulanic acid is significantly associated with a risk of DILI almost double compared to amoxicillin alone. The corresponding incidences are very low ( 0.1 cases for amoxicillin alone and 0.4 cases for amoxicillin +clavulanic acid for 100.000 inhabitants year, respectively), in line with other estimates from published data $[5,15,17,18]$. However, the true incidence of DILI is difficult to compute for several reasons; the most relevant ones are the following: the denominator of individuals receiving a drug is unknown, a simple objective test for the diagnosis of DILI is still lacking, and the causality of attribution to a single drug in patients exposed to many drugs is hard to determine [19].

The risk of co-amoxiclav induced DILI is known to be linked to clavulanic acid, since its association with other beta-lactam antibiotics has shown some hepatotoxicity $[20,21]$.

The amoxicillin component of the drug is primarily excreted by renal system, whereas clavulanic acid is excreted by both the kidney and liver. The mechanism of co-amoxiclav induced hepatitis is not completely clarified, but the frequent association with hypersensitivity reactions (i.e. skin rash, hypereosinophilia, anti-tissue antibodies) suggests an immune-allergic mechanism $[22,23]$. The reaction could be due also to an idiosyncratic response to the drug [12]. Moreover, currently available evidence suggests a genetic susceptibility to coamoxiclav induced liver injury in patients with specific HLA haplotype $[24,25]$.

Our findings, even though resulting from a small sample of observations, show a gender difference in risk of DILI associated with amoxicillin and co-amoxiclav. The observed higher risk for coamoxiclav in men is consistent with published data, such as those of Gresser [26] showing that 106 out of 153 cases of hepatitis were men.

Concomitant multi drug therapy is an acknowledged risk factor for DILI, and our findings show that cases associated with both amoxicillin or co-amoxiclav were exposed to a higher number of drugs than controls. According to Yazici et al. [27], the risk of death among users of co-amoxiclav increases with the use of concomitant hepatotoxic medications and they suggest that these patients may have concomitant diseases with less ability to recover from a severe DILI compared to healthier patients, or even that the occurrence of drug- 
drug interactions between amoxiclav and other hepatotoxic agents may result in a more serious liver injury. In their recent paper Suzuki et al. [28] analyzed the reporting frequency of liver events of four drugs (one of them was co-amoxiclav) in the presence of co-reported medications on the basis of the WHO global individual case safety report database $\left(\right.$ VigiBase $\left.^{\mathrm{Tx}}\right)$; they showed that co-reported drugs were associated with changes in the frequency of hepatic ADR reporting of drugs usually associated with hepatotoxicity, suggesting that co-medications could modify drug hepatic safety.

Our findings are also consistent with a published pharmacovigilance analysis from the Italian spontaneous reporting system database by Salvo et al. [13], which highlighted that co-amoxiclav use was associated with a higher frequency of liver injuries compared with amoxicillin alone.

Although the absolute risk of co-amoxiclav-induced DILI is generally very low, as in the case of the present work, it must be also interpreted in relation to the widespread use of the drug, which continuously increased over the years. In Italy, co-amoxiclav is the most prescribed antimicrobial agent (41\% of total antibiotics use) and among the most prescribed drugs [29], both for adults and pediatric patients. In the period 2007-2015 co-amoxiclav use increased from 7.3 to $9.4 \mathrm{DDD} / 1000$ inhabitants/day, whereas amoxicillin use decreased from 3.9 to 2.1 .

These evidence raise concern regarding the inappropriate prescribing of co-amoxiclav in particular for respiratory tract infections, for which several international guidelines still recommended amoxicillin as first line antibiotic treatment [30]. According to Butler et al. [31], antibiotic prescriptions virtually never benefit patients with acute cough, which is instead one of the most common reasons for antibiotic prescribing in the community. In Italy the prevalence of inappropriate use of antibiotics in upper respiratory tract infections has been estimated as $37.1 \%$ [29]. Another aspect suggesting a certain degree of inappropriateness in the use of penicillins for infections of the respiratory tract is represented by the seasonal variation in the use of such drugs. In fact, the Italian Report on the use of antibiotic drugs in 2009 [32] showed a correlation between the monthly incidence of influenza cases and antibiotic consumption. The treatment of disorders of viral origin with antibiotics is not only inappropriate, but can also substantially contribute to the development of antibiotic resistance.

The limitations and advantages of the main study have already been declared [14]. Among the others, the retrospective nature of the survey (case-control design), with the consequent difficulties to establish the temporal relationship between exposure and disease, as well as the difficulty of patients surveyed recall events that occurred long time before. Moreover, in this manuscript we reported the results of a subgroup of patients exposed to amoxicillin and co-amoxiclav, with all the limitations of the subgroup analysis [33,34], but it should be noted that this analysis was a pre-specified secondary endpoint in the study protocol. Among the strengths we find the methods for measuring consumption and possible damage due to amoxicillin and coamoxiclav, performed by direct administration of questionnaires to patients, who provided information collected and validated by each clinical monitor involved in the study. In addition, the data obtained are representative of the whole Italian population, having involved patients of Northern, Central and Southern Italy.

In conclusion, compared to amoxicillin alone, amoxicillin +clavulanic acid doubles the risk of serious acute liver injury, in agreement with most studies on DILI [15]. The incidence of coamoxiclav induced DILI is very low, however given the widespread use of this drug in the general population, the risk should be considered clinically relevant. The very often inappropriate prescription of antimicrobial agents, and in particular of co-amoxiclav, could expose a given patient to a life-threatening risk compared to a negligible clinical benefit.

\section{Acknowledgment}

We would like to thank Sara Grandi, PharmD, who actively participated in this research when preparing her graduation thesis at the Unit of Pharmacology, University of Bologna. This study was funded by the Italian Medicines Agency (AIFA), through a call for independent research (ID Study. FARM8B2TY7). The study was approved by the Independent Ethics Committee (IEC) of Verona Hospital (principal investigator and coordinating centre) and consecutively notified to the IECs of participating centres. Informed consent was obtained from all individual participants included in the study.

\section{References}

1. Versporten A, Coenen S, Adriaenssens N, Muller A, Minalu G, et al ESAC Project Group (2011) European surveillance of antimicrobial consumption (ESAC): outpatient penicillin use in Europe (1997-2009). J Antimicrob Chemother 66: vi13-23.

2. Andrade RJ, Lucena MI, Fernández MC, Pelaez G, Pachkoria K, et al. (2005) Drug-induced liver injury: an analysis of 461 incidences submitted to the Spanish registry over a 10 -year period. Gastroenterology 129 : 512-521.

3. Chalasani N, Fontana RJ, Bonkovsky HL, Watkins PB, Davern T, et al. (2008) Causes, clinical features, and outcomes from a prospective study of drug-induced liver injury in the United States. Gastroenterology 135: 1924-1934.

4. Bjornsson ES, JonassonJG (2013) Drug-induced cholestasis. Clin Liver Dis 17: 191-209.

5. Cundiff J, Joe S (2007) Amoxicillin-clavulanic acid-induced hepatitis. Am J Otolaryngol 28: 28-30.

6. Beraldo DO, Melo JF, Bonfim AV, Teixeira AA, Teixeira RA, et al. (2013) Acute cholestatic hepatitis caused by amoxicillin/clavulanate. World J Gastroenterol 19: 8789-8792.

7. Bessone F, Hernandez N, Lucena MI, Andrade RJ (2016) The Latin American DILI registry experience: A successful ongoing collaborative strategic initiative. Int J Mol Sci 17: 313.

8. Bolzan H, Spatola J, Castelletto R, Curciarello J (2000) Intrahepatic cholestasis induced by amoxicillin alone. Gastroenterol Hepatol 23: 237-239.

9. RuizRebollo ML, Aller De La Fuente R, Macho Conesa A, Salado Valdivieso I, Sainz Gil M, et al. (2011) Amoxicillin-induced cholestatic hepatitis. Gastroenterol Hepatol 34: 474-477.

10. Chaabane NB, Safer L, Njim L, Zakhama A, SaffarH (2011) Cholestatic hepatitis related to amoxicillin. Drug Chem Toxicol 34: 357-358.

11. Berg P, Hahn EG (2001) Hepatotoxic reactions induced by beta-lactamase inhibitors. Eur J Med Res 6: 535-542.

12. Rodríguez GLA, Stricker BH, Zimmerman HJ (1996) Risk of acute liver injury associated with the combination of amoxicillin and clavulanic acid. Arch Intern Med 156: 1327-1332.

13. Salvo F, Polimeni G, Moretti U, Conforti A, Leone R, et al. (2007) Adverse drug reactions related to amoxicillin alone and in association with clavulanic acid: data from spontaneous reporting in Italy. J Antimicrob Chemother 60: 121-126.

14. Donati M, Conforti A, Lenti MC, Capuano A, Bortolami O, et al. (2016) Risk of acute and serious liver injury associated to nimesulide and other 
Citation: Donati M, Motola D, Leone R, Moretti U, Stoppa G, et al. (2017) Liver Injury due to Amoxicillin vs. Amoxicillin/Clavulanate: A Subgroup Analysis of a Drug-Induced Liver Injury Case-Control Study in Italy. J Hepatol Gastroint Dis 3: 143. doi:10.4172/2475-3181.1000143

Page 5 of 5

NSAIDs: data from drug-induced liver injury case-control study in Italy. Br J Clin Pharmacol 82: 238-248.

15. Björnsson ES (2015) Drug-induced liver injury: an overview over the most critical compounds. Arch Toxicol 89: 327-334.

16. Bénichou C (1990) Criteria of drug-induced liver disorders: Report of an international consensus meeting. J Hepatol 1: 272-276.

17. Larrey D, Vial T, Micaleff A, Babany G, Morichau-Beauchant M, et al. (1992) Hepatitis associated with amoxycillin-clavulanic acid combination report of 15 cases. Gut 33: 368-371.

18. Larrey D (2002) Epidemiology and individual susceptibility to adverse drug reactions affecting the liver. Semin Liver Dis 22: 145-156.

19. Leise MD, Poterucha JJ, Talwalkar JA (2014) Drug-induced liver injury. Mayo Clin Proc 89: 95-106.

20. Van der Auwera P, Legrand JC (1985) Ticarcillin-clavulanic acid therapy in severe infections. Drugs Exp Clin Res 11: 805-813.

21. https://livertox.nih.gov/AmoxicillinClavulanate.htm

22. Dandakis D, Petrogiannopoulos C, Hartzoulakis G, Flevaris C, Lagoutari D, et al. (2002) Cholestatic hepatitis associated with amoxycillin clavulanic acid combination. A case report. Ann Gastroenterol 15: 85-87.

23. Kim SH, Saide K, Farrell J, Faulkner L, Tailor A, et al. (2015) Characterization of amoxicillin- and clavulanic acid-specific T cells in patients with amoxicillin-clavulanate-induced liver injury. Hepatology 62: 887-899.

24. Lucena MI, Molokhia M, Shen Y, Urban TJ, Aithal GP, et al. (2011) Susceptibility to amoxicillin-clavulanate-induced liver injury is influenced by multiple HLA class I and II alleles. Gastroenterology 141: 338-347.

25. Donaldson PT, Daly AK, Henderson J, Graham J, Pirmohamed M, et al. (2010) Human leucocyte antigen class II genotype in susceptibility and resistance to co-amoxiclav-induced liver injury. J Hepatol 53: 1049-1053.
26. Gresser U (2001) Amoxicillin-clavulanic acid therapy may be associated with severe side effects-review of the literature. Eur J Med Res 6: 139-149.

27. Yazici C, Mutlu E, Bonkovsky HL, Russo MW (2015) Risk factors for severe or fatal drug-induced liver injury from amoxicillin-clavulanic acid. Hepatol Res 45: 676-682.

28. Suzuki A, Yuen NA, Ilic K, Miller RT, Reese MJ, et al. (2015) Comedications alter drug-induced liver injury reporting frequency: Data mining in the WHO VigiBase ${ }^{\mathrm{mit}}$. Regul Toxicol Pharmacol 72: 481-490.

29. http://www.agenziafarmaco.gov.it/sites/default/files/ Rapporto_OsMed_2015_AIFA-acc.pdf

30. Woodhead M, Blasi F, Ewig S, Garau J, Huchon G, et al; Joint taskforce of the European respiratory society and European society for clinical microbiology and infectious diseases (2011) Guidelines for the management of adult lower respiratory tract infections--full version. Clin Microbiol Infect 17 Suppl 6: E1-59.

31. Butler CC, Hood K, Verheij T, Little P, Melbye H, et al. (2009) Variation in antibiotic prescribing and its impact on recovery in patients with acute cough in primary care: prospective study in 13 countries. BMJ 338: b2242.

32. http://www.agenziafarmaco.gov.it/sites/default/files/ Rapporto_sulluso_dei_farmaci_antibiotici.pdf

33. Fletcher J (2007) Subgroup analyses: how to avoid being misled. BMJ 335: 96-97.

34. Sun X, Briel M, Walter SD, Guyatt GH (2010) Is a subgroup effect believable? Updating criteria to evaluate the credibility of subgroup analyses. BMJ 340: c117. 